\title{
Morphometric and neurochemical alterations found in L-BMAA rats treated
}

Estefanía de Munck García ${ }^{a *}$, Emma Muñoz-Sáez ${ }^{b}$, Begoña G. Miguel ${ }^{b}, M^{a}$ Teresa Solas Alados ${ }^{c}$, Ana Martínez $^{\mathrm{d}}$, Rosa Ma Arahuetes $^{\mathrm{a}}$

a Departamento de Biología Animal II, Universidad Complutense de Madrid, 28040 Madrid, Spain

b Departamento de Bioquímica y Biología Molecular I, Universidad Complutense de Madrid, 28040 Madrid, Spain

c Departamento de Biología Celular (Morfología Microscópica), Universidad Complutense de Madrid, 28040 Madrid, Spain

d Centro de Investigaciones Biológicas, Consejo Superior de Investigaciones Científicas, 28040 Madrid, Spain.

E-mail addresses: e.munck@ucm.es (Estefanía de Munck García); emmamunoz@ucm.es (Emma Muñoz-Sáez); bgomezmi@pdi.ucm.es (Begoña G. Miguel); msolas@bio.ucm.es ( $\mathrm{M}^{\mathrm{a}}$ Teresa Solas Alados); ana.martinez@csic.es (Ana Martínez); rportero@bio.ucm.es (Rosa Ma Arahuetes).

*Corresponding author at: Fisiología Animal II, Universidad Complutense de Madrid, 28040 Madrid, Spain. Tel.: +34 652886457, E-mail address: estefania_nemesis@hotmail.com; e.munck@ucm.es. 


\section{Abstract:}

Amyotrophic Lateral Sclerosis (ALS) is a neurodegenerative disease characterized by progressive muscle paralysis that reflects the motoneurons' degeneration. Several studies support the relationship between $\beta-N$ methylamino-L-alanine (L-BMAA), a neurotoxic amino acid produced by cyanobacteria and diatoms, and the sporadic occurrence of ALS and other neurodegenerative diseases. Therefore, the study of its neurotoxicity mechanisms has charged great relevance in recent years.

Recently, our research group has proposed a model of ALS in rats by administration of L-BMAA that presents a number of pathophysiological features of ALS in humans. In this paper, we deepen the characterization of this model corroborating the occurrence of alterations present in ALS patients: decreased muscle volume, thinning of the motor cortex, enlarged lateral ventricles of the brain, alteration of both bulbar nuclei and neurotransmitters levels.

Keywords: ALS; L-BMAA; rat; neurotransmitters; motor cortex; NMR.

Abbreviations:

CHESS: Chemically sHift Selective Saturation

ET: echo time

FID: free induction decay

FOV: field of view

FSE: fast spin echo

MRI: Magnetic Resonance Imaging

MRS: Magnetic resonance spectroscopy

NA: number of acquisitions

OVS: Outer Volume Suppression

PRESS: point-resolved spectroscopy sequence

PT1/PT7: post-treatment day 1/ post-treatment day 7

PTFE: polytetrafluoroethylene

RARE: relaxation enhancement

T2WI: T2-weighted image

TauT: taurine transporter

TR: repetition time 


\section{INTRODUCION}

\subsection{Amyotrophic Lateral Sclerosis}

Amyotrophic Lateral Sclerosis (ALS) is a disease characterized by progressive muscular paralysis reflecting the degeneration of motoneurons in the primary motor cortex, the corticospinal tracts, the brainstem and the spinal cord (Wijesekera and Leigh, 2009). Among ALS patients, most cases are sporadic and only $5-10 \%$ are familiar.

This illness may debut, mainly, in two clinical forms: the spinal onset, which has two thirds of the cases, and the bulbar onset (Wijesekera and Leigh, 2009). In spinal onset, symptoms are related to focal muscle weakness in the upper and lower limbs. Gradually, spasticity may lead to weak atrophied limbs. Patients with bulbar onset usually present dysarthria and dysphagia for solids and liquids. Limbs' symptoms can be developed almost simultaneously with the bulbar symptoms in these patients.

The degeneration of different neuronal groups in ALS affects the patients' wide variety of clinical symptoms, that can show signs of spastic paresthesia and hyperreflexia, typical of cortical motoneurons, dysphagia and dysarthria, characteristic of bulbar palsy, or weakness, fasciculations and progressive muscular atrophy, typical of the lesion of spinal motoneurons, or a combination of them all (Berciano et al., 2003; Kato et al., 2014; Provenzale, 2004).

Neural impairment in ALS leads to alterations in neurotransmitter systems, reflected in a phenomenon of neuronal hyperexcitability. In addition to this phenomenon, changes in neurotransmitter levels in ALS patients have been extensively studied, detecting altered levels of glutamate, GABA and taurine (Malessa et al., 1991; Perry et al., 1987).

\subsection{L-BMAA}

The $\beta$-N-methylamino-L-alanine (L-BMAA) is a non-protein and non-lipophilic amino acid, highly neurotoxic produced by most cyanobacteria (Banack et al., 2007; Cox et al., 2005) and diatoms (Jiang et al., 2014). This amino acid has been linked to the sporadic appearance of several neurodegenerative diseases such as the amyotrophic lateral sclerosis-Parkinson dementia complex (ALS- 
PDC), Alzheimer's disease (AD) and amyotrophic lateral sclerosis (ALS) (Holtcamp, 2012); therefore the study of its mechanisms of neurotoxicity has charged great relevance in recent years.

The first studies about this molecule were performed regarding the high incidence of ALS-PDC in Guam, which was estimated to be 50-100 times higher than the global rate among the local population Chamorro in 1954 (Arnold et al., 1953; Kurland et al., 1954 a, b; Mulder and Kurland, 1987). The study of the Cycas' seeds (Cycas micronesica Hill.), which were consumed by the Chamorros, led to the discovery in 1967 of the L-BMAA (Vega y Bell, 1967; Vega et al., 1968). Then, in 2002 it was hypothesized that this amino acid could accumulate in animals that consume Cycas (Cox and Sacks, 2002). Finally, it was reported that both L-BMAA is produced by cyanobacteria of Nostoc genus that live as symbionts within specialized coral-shaped roots of Cycas (Cox et al., 2003), and the amount of L-BMAA that accumulates within proteins through the food chain in Guam ecosystem, is much higher than that initially estimated given only the free amino acid (Murch et al., 2004).

Recently, Dunlop et al. (2013) have shown that L-BMAA may be misincorporated into human protein chains in place of L-serine. Therefore, in humans or animals exposed to L-BMAA there is an internal neurotoxic reservoir which is slowly released even for years or decades after its consumption, by the hydrolysis of proteins. This fact may explain the long latency presented by ALSPDC. As Rodgers (2014) points out in a major review about this topic, the entry of non-protein amino acids in the human food chain and its incorporation into proteins can significantly contribute to neurodegenerative damage.

The discovery of L-BMAA in ALS and AD patients, besides being found in low concentrations in a patient who died of Huntington's disease (HD), has led some authors to propose the hypothesis that the accumulation of L-BMAA is a factor in the development of several neurodegenerative diseases (Pablo et al., 2009). Therefore, there may be a gene/environment interaction, with the LBMAA triggering neurodegeneration in genetically susceptible individuals (Rodgers and Dunlop, 2011). 
Regarding to its pathophysiological mechanisms, it is known that L-BMAA reacts with carbon dioxide $\left(\mathrm{CO}_{2}\right)$ under physiological conditions to form a carbamylated adduct, the $\beta$-(N-carboxy-N-methyl)-amino-L-alanine (BMAA- $\beta$ $\mathrm{NCO}_{2}$ ) (Myers and Nelson, 1990), which has a high structural similarity to glutamate and was proposed as the main responsible for the excitotoxic activity of L-BMAA. This carbamylated adduct acts on glutamate receptors NMDA, AMPA and metabotropic glutamate receptors (mGluRs). Furthermore, it has been suggested that the different susceptibility of different neuronal types to this amino acid, may depend on the glutamate receptor subtypes that they express (Liu et al., 2010).

\subsection{ALS animal models}

There are several models of ALS with transgenic animals that recapitulate some features of this disease. At least 13 lines of transgenic mice have been developed, which express human SOD1 proteins linked to familial ALS, including mutant active and inactive enzyme, and mutants with C-terminal truncated and deficient in metals (Turner and Talbot, 2008), although there are some transgenic models focused on the mutation of other proteins, such as the wobbler mouse (Boillèe et al., 2003). The SOD1G93A transgenic mouse model was the first to be developed and is currently the most studied (Walker and Atkin, 2011).

Although these rodent models develop several ALS neuropathological features (muscular denervation, astroglial and microglial activation, loss of motoneurons), it remains to be demonstrated that the findings obtained in the mutant SOD model can be applied to other forms of familial or sporadic ALS (Robberecht and Philips, 2013). In particular, the mouse model over-expressing mutated human SOD1, has been criticized for its poor predictive value in terms of potential therapies.

It should be noted that although the majority of ALS cases are sporadic (approximately $90 \%$ of cases), so far there are only genetic animal models expressing mutations linked to familial ALS.

Following the hypothesis that L-BMAA may be involved in the development of neurodegenerative diseases, several authors have examined the effects of 
poisoning with this amino acid to some animal species reaching substantial differences in their results. Most of these studies were compiled in an excellent review conducted by Karamyan and Speth (2008). The first studies were carried out in mice and nonhuman primates (Nunn et al., 1987; Ross and Spencer, 1987; Spencer et al., 1987 a, b.). Although various motor symptoms were described, the high doses used were strongly criticized and symptoms in primates were acute or subacute only.

In spite of the several studies about the toxicity and effects of L-BMAA made so far, there are not succeeded in establishing a sporadic ALS animal model that was easily reproducible and shows the progressive worsening of the disease. Recently, we have proposed in our research group a model of ALS in rats by administration of L-BMAA (de Munck et al., 2013), which mimics many of the pathophysiological features of ALS in humans. In the present work we delve into the characterization of this model focusing on the possible morphometric changes detected by Magnetic Resonance Imaging (MRI) and neurotransmitter levels in animals treated with L-BMAA.

\section{MATERIALS AND METHODS}

\subsection{Experimental animals}

Assays were carried out using male rats of Rattus norvegicus, albino variety, Wistar strain, that were supplied by Harlan Interfauna Ibérica S.A. (Barcelona, Spain). Animals were kept at a constant temperature $\left(21 \pm 1{ }^{\circ} \mathrm{C}\right)$ on a reversed light/dark cycle with diets and water ad libitum (A04 commercial rodent diet, Panlab, Barcelona, Spain). The trials were approved by the Ethics Committee for Animal Experimentation of the Universidad Complutense de Madrid.

\subsection{L-BMAA treatment}

At weaning time (postnatal day 21) treated animals were intraperitoneally (i.p.) injected with $300 \mathrm{mg} / \mathrm{kg}$ of L-BMAA (L-BMAA hydrochloride, Sigma-Aldrich, Ref. B107) during 5 consecutive days. L-BMAA was dissolved in phosphate buffered saline (PBS), giving a similar volume to control animals.

\subsection{Neurological evaluation}


In order to assess long term evolution of symptoms in treated animals, the neurological tests were carried out as described by de Munck et al. (2013), once a week during 14 months.

\subsection{Magnetic Resonance Imaging (MRI)}

To check the possible damage that could have caused the administration of LBMAA in muscular and nervous tissue, magnetic resonance imaging (MRI) analysis was performed, in vivo and ex vivo, in 6 treated animals and their respective controls.

\subsubsection{In vivo MRI}

The acquisition of images from the brain and the hind limbs of the animals was performed using MRI. During the procedure, the animals were anesthetized with isoflurane (Isoba ${ }^{\circledR}$ vet, Schering-Plough, S.A.) to $1.5-2 \%$, and were maintained at $37^{\circ} \mathrm{C}$. A breathing sensor compatible with magnetic resonance imaging was used to control them.

The MRI experiments consisted of two-dimensional and three-dimensional T2WI. All the experiments were performed on a BIOSPEC BMT 47/40 (Bruker, Ettlingen, Germany) spectrometer operating a $4.7 \mathrm{~T}$, equipped with an $11.2 \mathrm{~cm}$ actively shielded gradient system, capable of $200 \mathrm{mT} / \mathrm{m}$ gradient strength and $80 \mu$ s of rise time. A $7-\mathrm{cm}$ birdcage radiofrequency coil was used for transmission and reception.

T2WI were acquired using a fast spin eco (FSE), rapid acquisition with relaxation enhancement (RARE) technique, with a repetition time $(T R)=1650$ $\mathrm{s}, \mathrm{RARE}$ factor $=16$, and interecho interval $=10 \mathrm{~ms}$, resulting in an effective echo time $(E T)=80 \mathrm{~ms}$, with a number of acquisitions $(N A)=2$. A field of view $(F O V)=3.5 \times 3.5 \times 1.75 \mathrm{~cm}^{3}$ were used in case of the images acquired from the brain, and a FOV $=8.0 \times 4.0 \mathrm{~cm}^{3}$ were used for the images acquired from the hind limbs. The acquired matrix size was $256 \times 256 \times 64$. These data were zero-filled to obtain a reconstructed matrix size of $256 \times 256 \times 256$.

T2WI image analysis was performed using ImageJ 1.33u (National Institutes of Health, Bethesda, MD). Brain areas analyzed were total cerebral cortex, the 
motor cortex (areas M1 and M2) and the lateral ventricles, which were selected according to the atlas of the rat brain (Paxinos and Watson, 2005). In the case of hind limbs, the total volume of skeletal muscle was measured. Regions of interest were manually drawn for each slice subsequently adding the total value obtained in each of them to obtain the total number of voxels. To calculate the total volume, the total number of voxels was multiplied by the volume of each voxel (previously calculated by multiplying the resolution obtained in the three dimensions of the voxel). Volumes obtained are finally represented as volume $\left(\mathrm{mm}^{3}\right)$ / total brain volume $\left(\mathrm{mm}^{3}\right) \times 100$ for the brain regions measured, and as muscle volume $\left(\mathrm{mm}^{3}\right) /$ total animal weight $(\mathrm{g})$ for the hind limbs, thus taking the weight of the animal as a reference to the total size.

\subsubsection{Ex vivo $M R I$}

Fourteen months-old animals, both treated and control, were sacrificed under general anaesthesia (ketamine $\left(100 \mathrm{mg} / \mathrm{ml}\right.$ ) (Imalgene $1000^{\circledR}$, Merial) / xylazine (2\%) (Rompun, Bayer)). Subsequently, they underwent a perfusion with a fixative solution of $10 \%$ formalin $(37-40 \%$ formaldehyde (Panreac) in a phosphate buffer). After the fixation, the whole brain of each animal was removed, keeping the tissue at $4^{\circ} \mathrm{C}$ in a solution of $10 \%$ formalin until image acquisition.

The MRI scanner used ex vivo was the same as the one used to obtain images in vivo but with a different gradient and birdcage systems: gradient system of 6 $\mathrm{cm}$ with a maximum gradient strength of $450 \mathrm{mT} / \mathrm{m}$, a rise time of 80 microseconds and a volume birdcage of $3 \mathrm{~cm}$ (Bruker). To carry out the MRI scanner, the brains were placed in Fluorinert FC-40 (3M, Minnesota, USA).

T2W images were acquired using a 3D sequence FSE with the following parameters: TR $=1.6395 \mathrm{~s}$; Effective ET $=72 \mathrm{~ms}$; FOV $=2.5 \times 2.5 \times 2.5 \mathrm{~cm}^{3}$, $N A=1$. The dimensions of the matrix acquisition and reconstruction were $256 x$ $192 \times 64$ and $256 \times 256 \times 64$, respectively.

\subsection{High-performance liquid chromatography (HPLC)}

In order to study neurotransmitter levels in the short term after treatment with LBMAA, two groups of 10 animals each and their respective controls were used. The animals were sacrificed under conditions of strong sedation with isoflurane 
(Isoba®vet, Schering-Plough, SA), $5 \%$ constant flow of $\mathrm{O}_{2}$, at two different posttreatment times: 24 hours after the last injection (PT1) and one week after the last injection (PT7). Mass brain was quickly removed, frozen in liquid nitrogen and stored at $-80^{\circ} \mathrm{C}$. After the extraction of the motor cortex, samples were maintaining in cold conditions to prevent degradation. Each sample was weighed and homogenized in $500 \mathrm{ml}$ of ultrapure $\mathrm{H}_{2} \mathrm{O}$, immediately centrifuged at $11000 \mathrm{rpm}$ for $10 \mathrm{~min}$ at $4^{\circ} \mathrm{C}$. Supernatants were collected with sterile syringes of $1 \mathrm{ml}$ (Omnifix®-F Braun) and filtered with filters PTFE (polytetrafluoroethylene) $0.45 \mu \mathrm{m}$ pore (OlimPeak ${ }^{\circledR}$ Teknokroma).

Samples were derivatized with OPA reagent (30 mM o-phthalaldehyde (SigmaAldrich), 0.75\% 3-mercapto-propionic acid (Sigma-Aldrich), 10\% methanol (HPLC grade, LabScam), in borate buffer (0.4M boric acid, Merk)) at pH 9.5. Determinations were performed by HPLC (Varian 920 LC) with fluorescence detector (excitation $\lambda$ of $365 \mathrm{~nm}$, emission $\lambda$ of $455 \mathrm{~nm}$ ) using a Nucleosil 100 C18 (150 x $4.6 \times 5 \mu \mathrm{m}$, Varian) column. To perform the calibration curves, a number of aminoacid standard solutions were prepared: glutamate (Sigma, ref G8415), GABA (Sigma, ref A2129) and taurine (Sigma, ref. T0625). From these solutions, calibration curves were obtained in a range of $0.2 \mathrm{ppm}$ to $1.4 \mathrm{ppm}$. The chromatograms were integrated using Varian software Galaxie ${ }^{\circledR}$, finally transforming the data plotted as $\mathrm{mg}$ of amino acid / $\mathrm{g}$ of tissue.

\subsection{Magnetic resonance spectroscopy (MRS)}

The study of the levels of neurotransmitters by magnetic resonance spectroscopy (MRS) in the motor cortex (figure 1), was carried out 5 months post-treatment (10 treated and 10 control rats) under the same conditions and with the same birdcage as the other in vivo MRI experiments (section 2.4.1). MRS was performed using a point-resolved spectroscopy sequence (PRESS) from an $8 \times 3 \times 5 \mathrm{~mm}^{3}$ voxel with TR/TE=3000/136 ms, NA=256, number of points $=4096$ and spectral bandwidth $=2003.21 \mathrm{~Hz}$. Shimming was performed over the spectroscopy voxel by adjusting the currents in the three linear and $z 2$ shim coils. Typically, the water linewidth was around $20 \mathrm{~Hz}$. For water signal suppression, CHESS pulses (Chemically sHift Selective Saturation) was used, saturating a spectral width of $60 \mathrm{~Hz}$ and using the option OVS (Outer Volume Suppression) with a width of $15 \mathrm{~mm}$. The FID (free induction decay) was zero- 
filled to 32000 points, multiplied by an exponential function with a $2 \mathrm{~Hz}$ line broadening, baseline corrected, Fourier transformed and phased. The spectra obtained were processed with TOPSPIN software, version 1.3 (Bruker Rheinstetten, Germany).
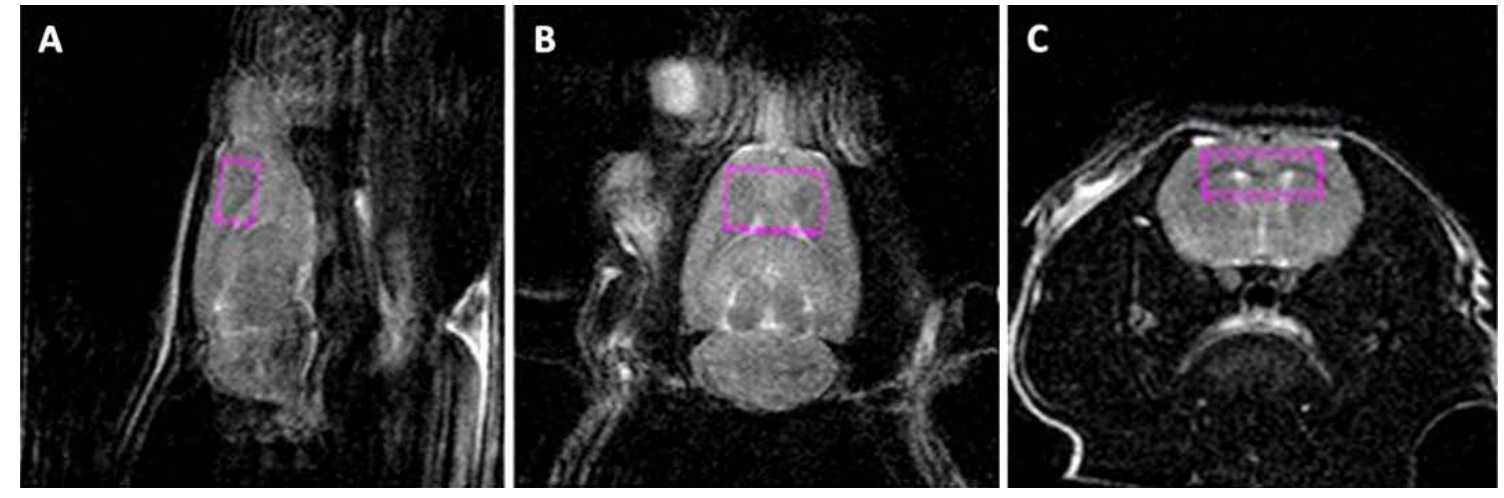

Figure 1: Representative images of selected area for the MRS study, in a sagittal (A), axial (B) and coronal (C) section.

\subsection{Statistical analysis}

The results are expressed in the graphs as mean \pm standard error of the mean (SEM). Data were analyzed using a t-test when only the groups of control and treated animals were compared (volume of the hind limbs and the brain regions and neurotransmitters at post-treatment level of the long term) and using oneway ANOVA when more than two groups were compared (neurotransmitters level short term post-treatment).

All analysis and data processing were performed with GraphPad Prism v 5.03. Differences were considered significant at a p-value $\leq 0.05$.

\section{RESULTS}

\subsection{Long-term neurological evaluation}

In figure 2 it is shown that the neurological evaluation of rat males treated with $300 \mathrm{mg} / \mathrm{kg}$ of L-BMAA for 5 consecutive days at weaning time, increases progressively during the period of study. Although during the first 3 months the evolution increase fast, then there is a stabilization phase in which the assessment increases more slowly. 


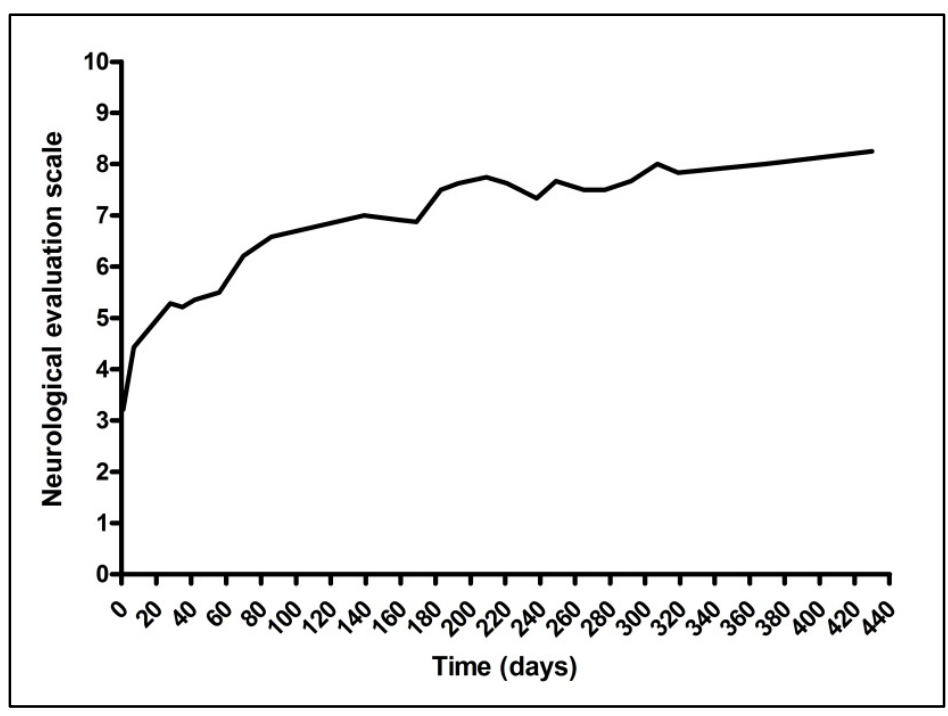

Figure 2: Neurological evaluation evolution during 14 months in male rats treated with $300 \mathrm{mg} / \mathrm{kg} /$ day of L-BMAA 5 consecutive days. Control animals evaluation is not shown because it coincides with abscise axis.

\subsection{Hind limbs muscular volume}

Analysis of muscular volume of the hind limbs by in vivo MRI revealed that there is a progressive loss of muscle volume in animals treated with L-BMAA compared to control animals. 6 months-old treated rats showed a significant decrease in muscle volume in the left hind limb $(t=3.186, p=0.033)$, with $a$ $17.5 \%$ of volume loss, whereas, the right hind limb showed no significant differences, although a $15 \%$ volume decrease is appreciated (figure 3A).

At 8 months of age (figure 3B), these animals show a greater loss of muscle volume in both hind limbs. The left hind limb shows a significant volume decrease $(t=3.186, p=0.021)$, arround $24 \%$ of volume loss, while in the right hind limb there is a significant volume decrease $(t=3.281, p=0.031)$, of almost $20 \%$. 


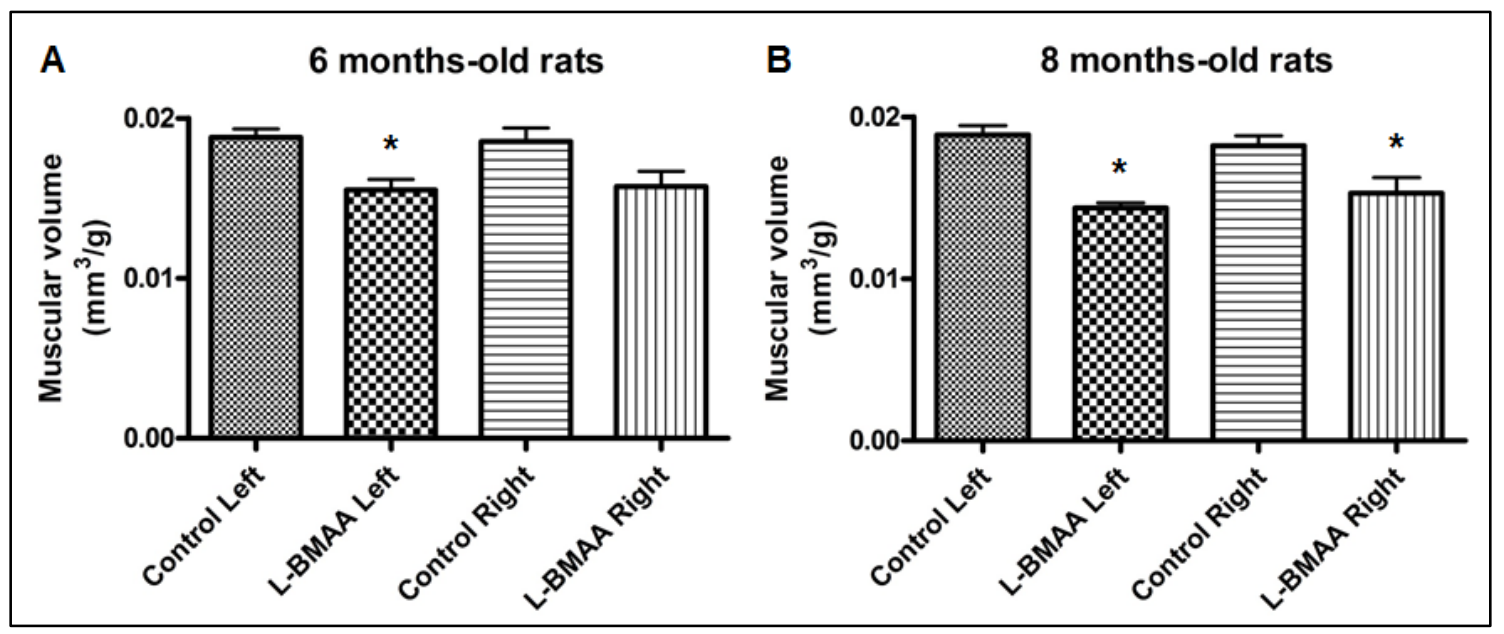

Figure 3: Muscular volume of the hind limbs relative to the animals' weight, $A$ ) at 6 months of age (5 months post-treatment) and B) at 8 months of age (7 months after treatment). * Indicates differences p-value $<0.05$ to the control group.

\subsection{Brain MRI study}

In order to check for possible alterations through the motor pathway, magnetic resonance imaging (MRI), a technique of $3 \mathrm{D}$ image that has been used in morphometric studies for the quantification of the volume of gray matter and white matter in the central nervous system, was used (Ashburner and Fristone, 2000; Kollewe et al., 2012).

\subsubsection{Volume of the cerebral cortex}

The analysis of changes in volume of the cerebral cortex by in vivo MRI revealed that, at 8 months of age ( 7 months after treatment), treated animals showed no significant differences in the total volume of cerebral cortex of both hemispheres (figure 4A) compared to their respective controls. However, significant differences in the volume of the motor cortex (M1 and M2 areas) are observed. Significant decreased volume is observed in the right motor cortex $(\mathrm{t}$ $=5.28, p=0.0061)$ of $10.28 \%$, and in the left motor cortex $(t=4.965, p=$ 0.0077 ) (figure $4 \mathrm{~B}$ ) with a decrease of $18.77 \%$ in animals treated with L-BMAA compared to controls. 


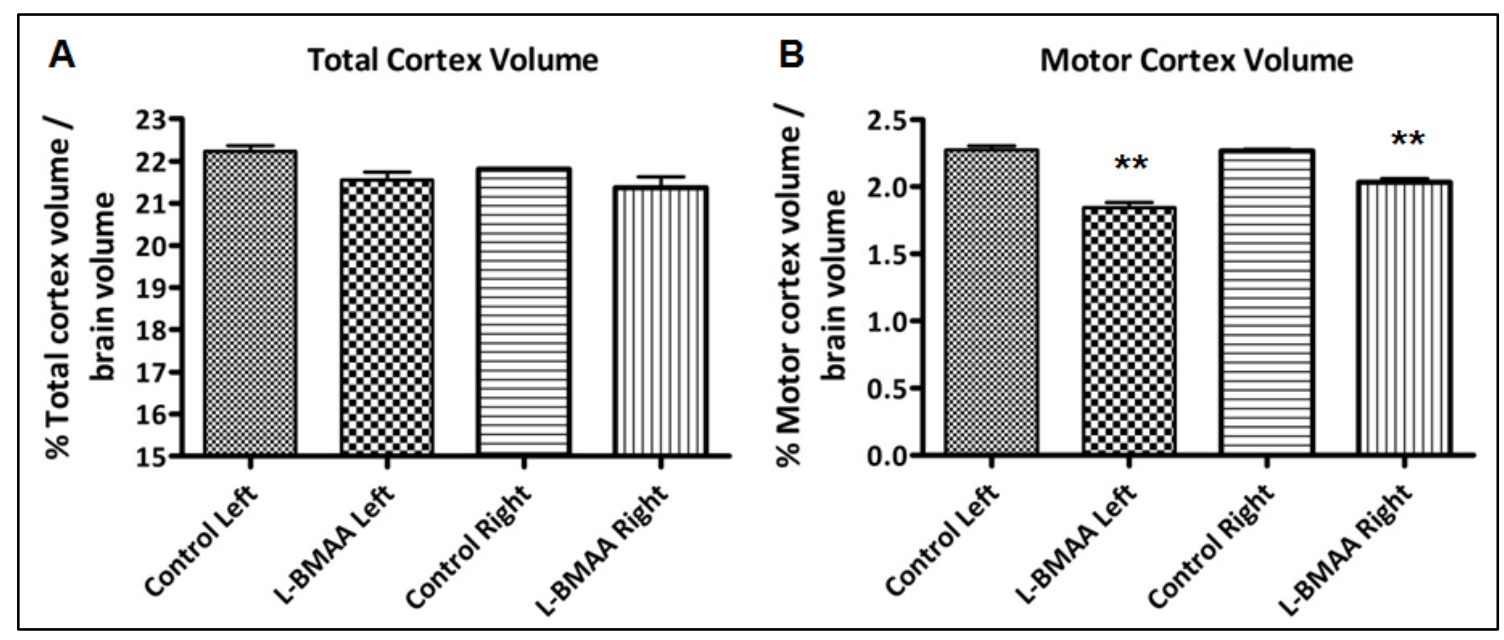

Figure 4: Volume percentage of both, the cerebral cortex $(A)$ and the motor cortex $(B)$, relative to the total volume of the brain. ** Indicates differences $p$ value $<0.01$ to the control group.

\subsubsection{Volume of the lateral ventricles}

MRI images taken in vivo from 8 months old rats (7 months after treatment) showed an increased volume of the lateral ventricles in treated animals (figure 5). However, subsequent processing of the images showed no significant differences in right or left ventricles, although a tendency to increase the volume of them was shown (figure 6). Should be noted that the increased volume of the right lateral ventricle is $59.39 \%$ and the left is $27.65 \%$ with respect to controls. The absence of significance in the statistical analysis would exist because these data show great variability. 

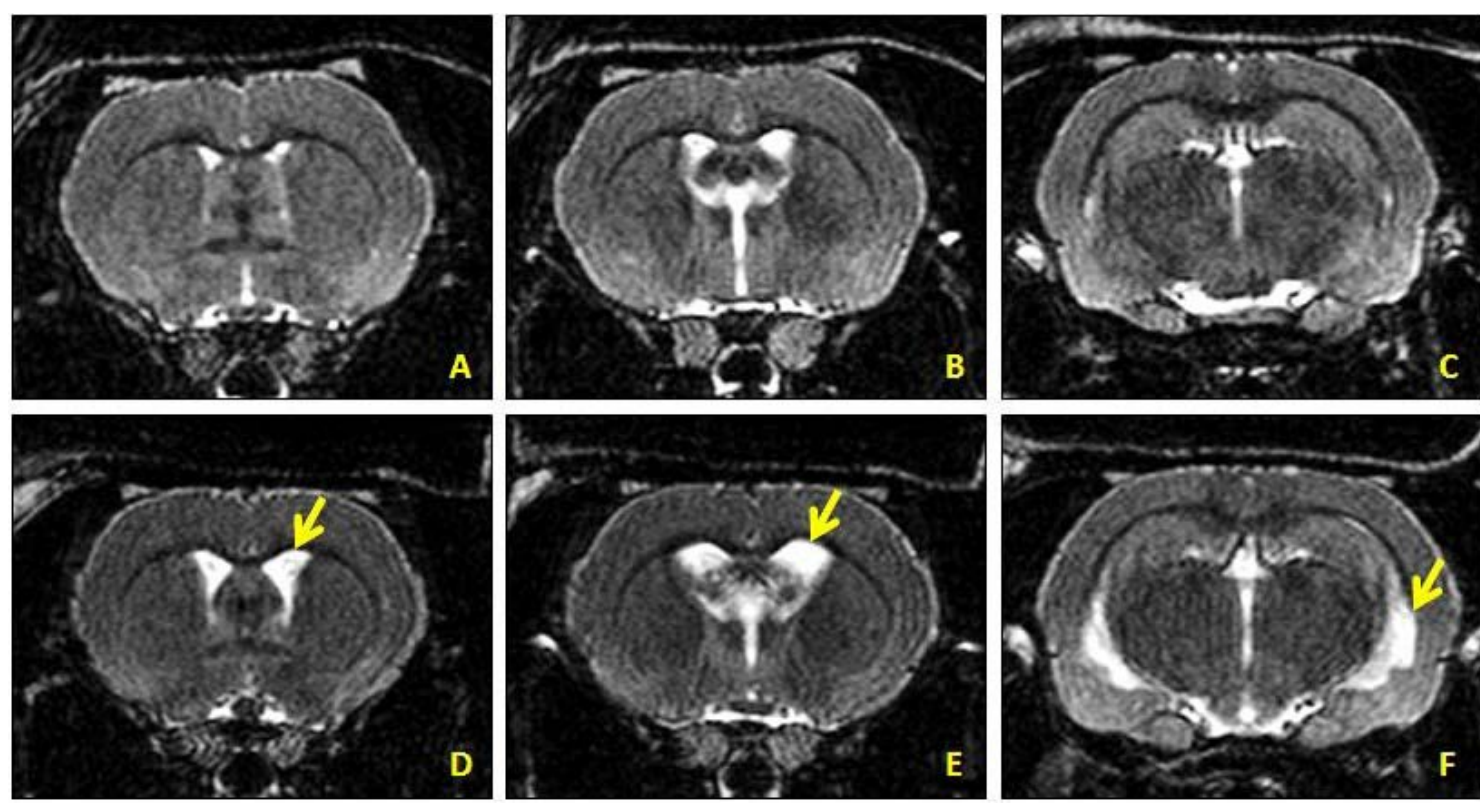

Figure 5: MRI from the brain of both, a representative control animal (images $A$, B and C) and a representative treated animal (images D, E and F), eight months after the end of the treatment. Large differences in the volume of the lateral ventricles (marked with arrows) between control and treated animals can be appreciated.

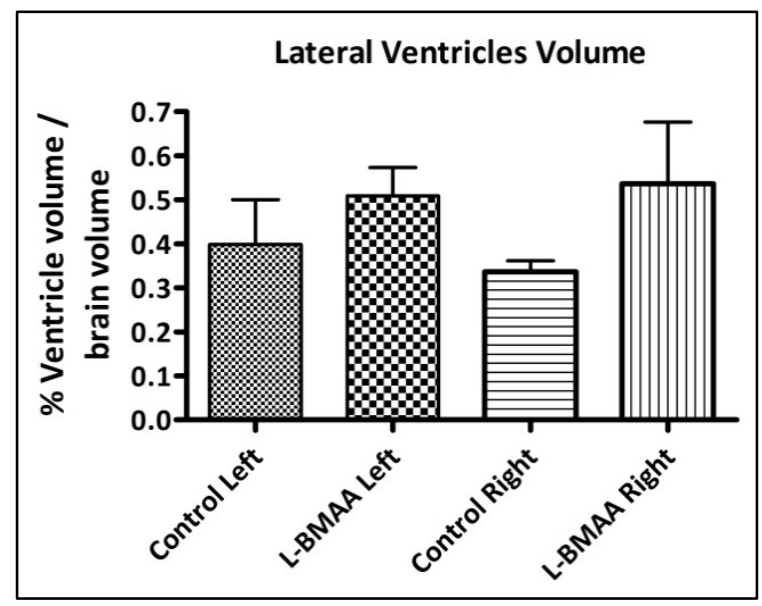

Figure 6: Percentage of volume of the lateral ventricles relative to the total brain volume. 


\subsubsection{Altered bulbar nuclei}

Through MRI obtained ex vivo, differences in intensity throughout the brainstem between treated and control rats were analyzed. Our results show a clear increase in the intensity of the trigeminal motor nucleus and the solitary tract nucleus (figure 7), both involved in the swallowing process, in animals treated with L-BMAA compared to control animals.
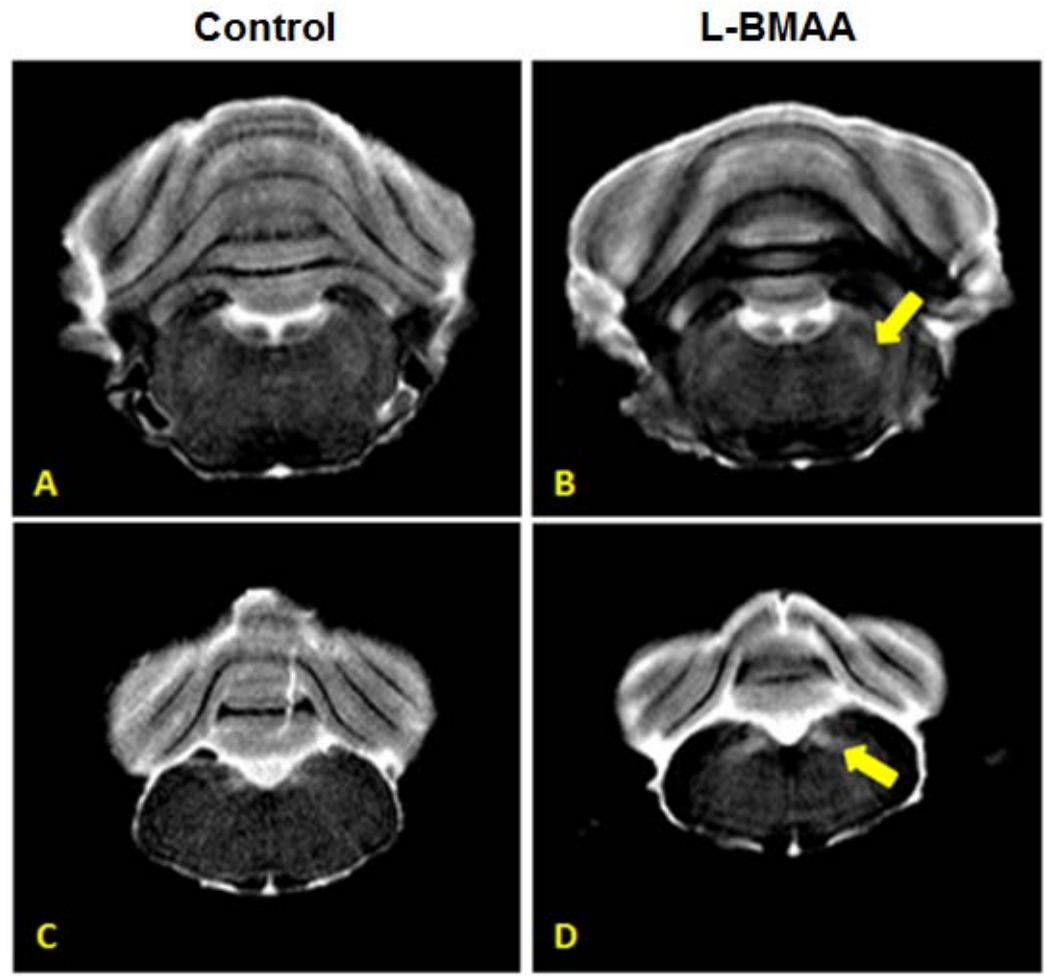

Figure 7: Magnetic resonance imaging ex vivo at bulbar level, representative of the observed changes between the control group and the treated group. A-B) Pictures took at level of the motor trigeminal nucleus of control and treated animals, respectively. C-D) Pictures at level of the solitary tract nucleus of control and treated animals, respectively. Hyperintensities found in the treated animals are indicated by arrows.

\subsection{Neurotransmiters alteration in the motor cortex}

In order to study the neurochemical changes caused by treatment in vivo with LBMAA in the motor cortex, two diferent tests were conducted: on the one hand, neurotransmitter levels were measured in the short term after the end of treatment by HPLC, and on the other hand, to determine whether there are alterations in neurotransmitter systems in the long term, these levels were 
measured by magnetic resonance spectroscopy. Although HPLC provides more accurate measurements, long term analisys were carried out with magnetic resonance spectroscopy to prevent the slaughter of animals and to continue the study about their evolution in the longer term.

\subsubsection{Short-term neurochemical alterations}

In short-term assays, rats treated with L-BMAA one day post-treatment (PT1) and seven days post-treatment (PT7) and their respective controls were used. Control groups at PT1 and PT7 showed no significant difference between them, so that only one control group was represented in figure 8 to simplify illustration.

Glutamate levels (figure 8A) were elevated in the motor cortex of treated animals $(F=49.36, p=0.0005)$. The post-test revealed that significant increase in the concentration of glutamate at PT1 ( $\mathrm{t}=8.120, \mathrm{p}<0.01)$ and highly significant increase at PT7 ( $t=8.752, p<0.001)$ occurs. There were no significant differences between animals treated in the two post-treatment time points

GABA levels were significantly decreased by treatment with L-BMAA (F = 24.70, $p=0.0026$ ) (figure 8B). The post-test showed that the concentration of GABA was significantly less on both PT1 $(\mathrm{t}=4.942, \mathrm{p}<0.05)$ and PT7 day $(\mathrm{t}=$ 6.964, $p<0.01$ ). There were no significant differences in GABA levels between the two post-treatment days.

Taurine concentration was significantly higher in the motor cortex $(F=0.0007, p$ = 44.44) (Figure 8C). In PT1 day, treated animals showed a highly significant increase compared to controls $(t=9,421, p<0.001)$ whereas in the PT7 day the increase is significant $(t=4.532, p<0.05)$. Taurine levels were significantly higher at PT1 than PT7 $(t=3.894, p=0.05)$. Therefore, there was an increase in taurine levels in response to treatment with L-BMAA that remained elevated one week after the treatment, but less than at the end of treatment. 


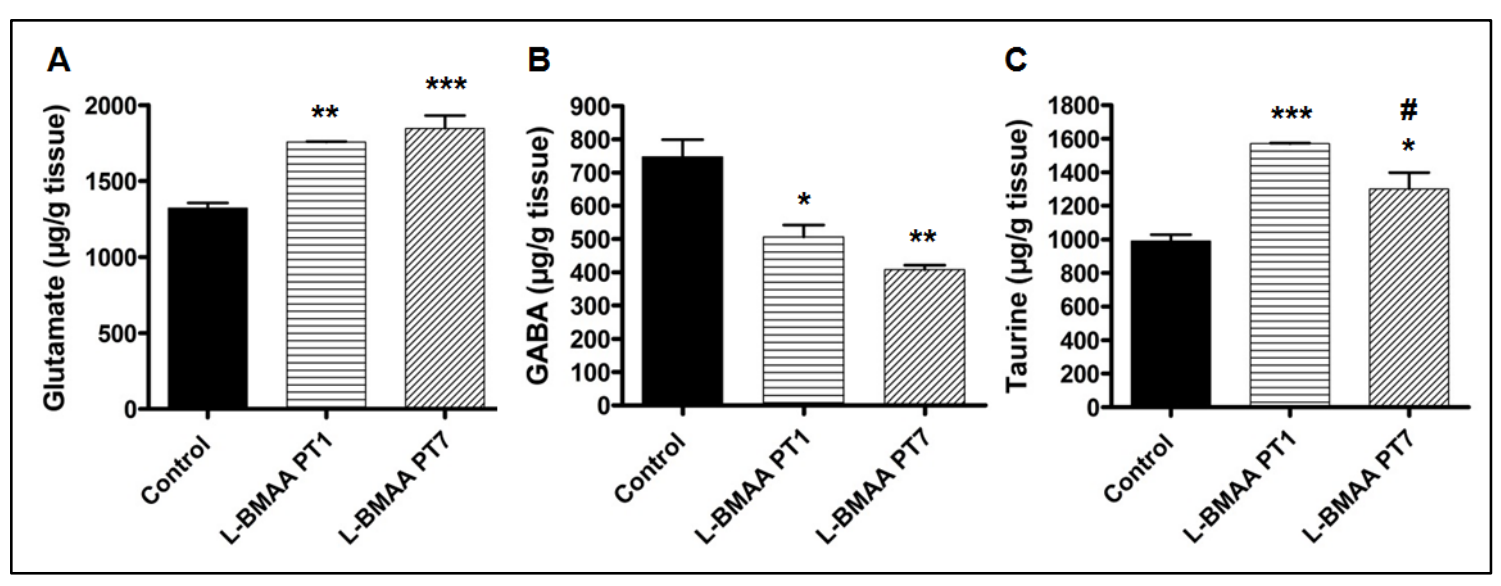

Figure 8: Neurotransmitters concentration ( $\mu \mathrm{g} / \mathrm{g}$ tissue) in the motor cortex of control animals and animals treated with L-BMAA one day after treatment (PT1) and 7 days after treatment (PT7). A) Concentration of glutamate. B) Concentration of GABA. C) Concentration of taurine. *, **, *** Indicate differences p-value $<0.05<0.01<0.001$ with the control group, respectively. \# indicates differences $p$-value $<0.05$ between L-BMAA treated animals in the day PT1 and PT7.

\subsubsection{Long-term neurochemical alterations}

In the motor cortex of treated animals, 5 months after treatment, glutamate concentration was significantly higher than in control animals $(p=3.986, p=$ 0.0163) (figure 9A), while levels of GABA were highly significantly lower $(t=$ 10.17, $p=0.0005$ ) (figure 9B). Although no significant difference in the concentration of taurine relative to the control group was observed, a trend is seen to stay higher in treated animals (figure 9C).

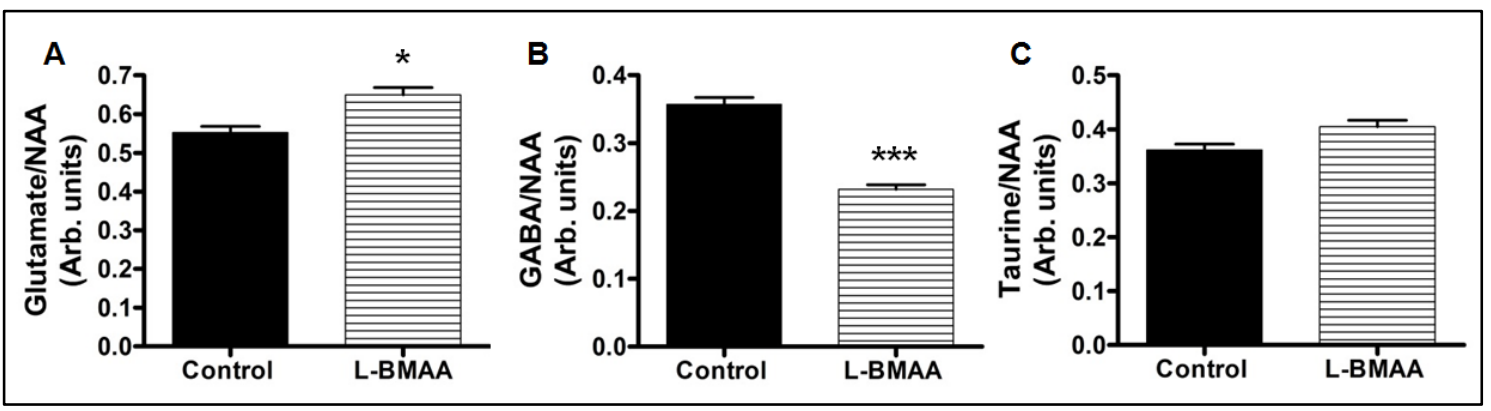

Figure 9: Level of neurotransmitters in the motor cortex of control animals and animals treated with L-BMAA, at 5 months posttreatment. The assessment is indicative of the level of NAA and expressed in arbitrary units (Arb. units). A) Concentration of glutamate; B) Concentration of GABA; C) Concentration of 
taurine. * ${ }^{* \star *}$ Indicate $\mathrm{p}$-value differences $<0.05<0.001$ with the control group, respectively.

\section{DISCUSSION}

\subsection{Neurological evaluation}

The observation of long-term neurological evaluation (figure 2) shows that there are several phases in the evolution of symptoms in the treated animals. First, a stage of rapid development occurs during the first three months after treatment, possibly due to excitotoxicity caused by L-BMAA in the central nervous system, which is consistent with that observed by other authors (Ross et al., 1987; Lobner et al., 2007). Then, a second stabilization stage in which symptoms develop more slowly occurs, possibly due to a gradual release of the reservoirs of L-BMAA in brain. This observation is consistent with the explanation given by Murch et al. (2004) for the late onset of ALS-PDC among the Chamorro.

\subsection{Morphometric changes in MRI study}

This study has revealed that animals treated with L-BMAA present a progressive decrease in muscle volume in the hind limbs that could involve muscular atrophy, being indicative of a loss of innervation from the motoneurons of the spinal cord. The muscle atrophy is consistent with results obtained in previous studies with rats treated with this dose of L-BMAA (Munck et al., 2013), which describes a loss of motor control, analyzed by functional tests, which may be due to alterations in several points of the motor pathway.

In ALS cases, particularly in patients with spinal disease onset, it has been observed focal muscle atrophy in early testing, in the upper limbs and lower limbs (Wijesekera and Leigh, 2009). These clinical features are indicative of the loss of neurons at all levels of the motor system, from the cortex to the anterior horn of the spinal cord (Mitchell and Borasio, 2007).

By means of this volumetric study, it has been shown a decrease in the motor cortex volume in animals treated with L-BMAA compared to control animals. In contrast, no differences in the total volume of the cerebral cortex were found, indicating that there is a much focused damage on specific regions of the cortex involved in movement control. It is important to note that this occurs concomitantly with a huge increase in the volume of the lateral ventricles, of 
almost $60 \%$ in the right lateral ventricle and over $27 \%$ in the left lateral ventricle (figure 6).

Several studies have reported volumetric atrophy of primary motor cortex in patients (Kollewe et al., 2012) but, surprisingly, there is no consensus in all the papers published so far (Agosta et al., 2007; Filippini et al., 2010; Grosskreutz et al., 2006; Mezzapesa et al., 2007). Recently, Mezzapesa et al. (2013) have confirmed the reduction in volume of the primary motor cortex in ALS patients compared with healthy controls while Cosottini et al. (2013) described the gray matter atrophy in motor and extra-motor areas of the cortex. This volume loss can be explained by Nishihira et al. (2008) studies, which describes severe neuronal loss evident in the upper and lower motoneurons' systems in analysis of samples of sporadic ALS patients.

Other works combine morphometric studies with diffusion studies (diffusion tensor imaging - DTI) to check the alterations in white matter tracts. Using this technique it has been found in ALS patients alterations involving rostral corticospinal tract and corpus callosum (Filippini et al., 2010; Prell et al., 2013). Recently, it has been found that these changes, along with the thinning of the primary motor cortex, correlate with the progression of the disease (Lillo et al., 2012; Verstraete et al., 2010).

Importantly, Kolind et al. (2013) found in patients a significant demyelination in the brain and spinal pyramidal tract. They investigated these degenerative processes using a new MRI sequence (mcDESPOT) sensitive to water pools in myelin and in the intracellular and extracellular spaces. Thus, they showed that ALS patients show an increase of intracellular and extracellular water, a sign indicating neuroinflammatory activity.

Neuroinflammation has been established as an important factor in the pathogenesis of many neurodegenerative diseases, including ALS. At various points along the course of the disease both, astrocytes and microglia appear markedly activated, causing neuroprotective and pro-inflammatory molecules, which may decrease or increase the rate of degeneration of primary motoneurons, respectively (Evans et al., 2013). There is a significant activation 
or proliferation of microglia and astrocytes in specific stages of the disease in transgenic mouse models of ALS (Hall et al., 1998; Weydt et al., 2002) that have also been observed in humans in vivo (Turner et al., 2004) and in post mortem examinations (Anneser et al., 2004). Andjus et al. (2009) found a patent dilatation of the lateral ventricles in a genetic rat model of ALS, which was related to neuroinflammatory processes, which were possibly facilitated by the presence of a compromised BBB.

Although our study has not been proven activation of the immune system, signs of neurodegeneration, i.e., decreasing volume of the motor cortex and, above all, the remarkable increase in ventricular volume that represents a significant increase in extracellular water, lead us to think that this type of neuroinflammatory processes have been activated.

\subsection{Bulbar nuclei alterations}

Routine diagnostic examinations using MRI in humans are mainly aimed at the detection of neuronal degeneration, which is seen as hyperintensities on MRI (Andjus et al., 2009). Although motor deficits typically predominate in the limbs, bulbar innervation can also be severely affected, leading to atrophy of the tongue, dysphagia and dysarthria (Guegan and Przedborski, 2003). There are evidences of a differential vulnerability among motoneurons, being certain motor nuclei of cranial nerves typically affected in ALS, as the trigeminal, facial, hypoglossal and ambiguous (Reiner et al., 1995).

Rats treated with L-BMAA in our experimental model show clear intensity increase in the motor trigeminal nucleus and the nucleus of the solitary tract, in the images taken by MRI ex vivo. The nucleus of the solitary tract is an important center for afferent visceral information. Among other functions receives direct sensory stimulus taste buds and is a major control center of the autonomic nervous system. It also receives visceral sensory stimuli of the vagus nerve, so that this nucleus is a key center of integration in the control of feeding and metabolism. On the other hand, the motor trigeminal nucleus is responsible for the efferent innervation of the muscles of mastication, the mylohyoid and palate tensor involved in the process of chewing and swallowing. Both nuclei are involved in the neural control of swallowing and throwing up. 
Our results agree with those obtained in ALS genetic models in which it is described a foci of neurodegeneration in the brainstem, in areas corresponding to the motor trigeminal nucleus and the vagus nuclei, the spinal trigeminal tract and nucleus ambiguous among others (Andjus et al., 2009; Angenstein et al., 2004; Bataveljić et al., 2009; Bucher et al., 2007; Niessen et al., 2006; Zang et al., 2004). In addition, MRI hyperintensities were found in similar regions of the brainstem of patients with ALS (Basak et al., 2002, Hecht et al., 2002, Zhang et al., 2003).

Moreover, the involvement of the bulbar nucleus in the later stages after the treatment in which problems have not yet observed in swallowing, implies that these animals developed more type of pathology similar to that present in patients with ALS debut cord, where the disease present with symptoms in the limbs and only in the final stages bulbar symptoms develop.

\subsection{Impaired neurotransmiters systems}

As indicated by Bae et al. (2013), there is an open debate on the origin of ALS, but there is now growing support for the theory that hyperexcitability develops due to the cortical origins of ALS. Hyperexcitability in ALS appears to be developed in a closely related manner with the affected interaction between two different parts of the human neocortex, projection neurons (excitatory) and interneurons (inhibitory) interaction (Bae et al., 2013).

The precise mechanisms that lead to the death of motoneurons in ALS are still unknown, although it is generally accepted that excitotoxicity plays a role, as the primary action or as an indirect consequence of oxidative stress (Leigh and Meldrum, 1996; Robberecht, 2000). However, different results have been obtained in several studies regarding the levels of neurotransmitters in the analysis of different samples of patients, possibly due to the heterogeneity of the disease and/or the different methodologies used to analyze the content of excitotoxic aminoacids (Dunlop et al., 2013). Spreux-Varoquaux et al. (2002) detected high concentrations of glutamate in the cerebrospinal fluid of patients with ALS, also correlated with both the spinal disease onset, more impaired function of the limbs and a higher rate of muscle deterioration. 
Our results show that there are increased levels of glutamate in the motor cortex at the end of treatment with L-BMAA, which increases one week later (figure 8A) and remain high 5 months after treatment (figure 9A). This fact involves a long-term damage on the glutamatergic system or a loss of depolarization control in the glutamatergic neurons. It could indicate that LBMAA can cause excitotoxic processes, which remain long time after the end of the treatment. Moreover, it correlates with the volume loss in the motor cortex detected. These findings are consistent with the detection of damaged mitochondria in nervous tissue of rats treated with L-BMAA (Munck et al., 2013), suggesting that excitotoxic mechanisms might play a role in the pathogenesis developed due to the action of this neurotoxic.

The glutamate increase detected may also be due to the actions of L-BMAA on xc- system, including increased glutamate release into the extracellular medium, and which have been directly related to the neuronal death induced by L-BMAA (Fogal et al., 2007; Liu et al., 2009; Murphy et al., 1989, 1990). The glutamate-induced excitotoxicity can cause neurodegeneration through activation of calcium-dependent enzyme pathways (Bae et al., 2013), and by the increased production of free radicals, which can cause neurodegeneration through damage to organelles and upregulation of proinflammatory mediators (Hensley et al., 2006). Both excitotoxicity and glutamate defective metabolism have been implicated in the pathogenesis of ALS (Andreadou et al., 2008).

The motoneuron excitability is tightly regulated by presynaptic inhibition mediated by glycinergic and GABAergic innervation. GABA release is a function of interneurons (that is $20-25 \%$ of the neocortical neurons). Despite the findings in ALS suggest that must be affected, the integrity of the inhibitory systems that regulate motoneurons has not been sufficiently studied (Martin and Chang, 2012). Physiologically, GABAergic transmission in the cortex has been found to provide a protective effect of excitatory projection neurons (Bae et al., 2013).

Importantly, in addition to the hyperexcitability of the projection neurons, the weak inhibitory GABAergic transmission has been documented in electrophysiological and pathological studies in patients with ALS. Nihei et al. (1993) demonstrated that GABAergic inhibitory neurons were reduced in the 
motor cortex of ALS patients compared to healthy subjects. Recently, Foerster et al. $(2012,2013)$ showed a decrease in the content of GABA in the motor cortex of these patients, by using magnetic resonance spectroscopy techniques.

Decreased GABA levels detected in this study, both short term and long term (figures $8 \mathrm{~B}$ and 9B) implies a loss of GABAergic signaling in the motor cortex that would worsen with the neurological symptoms in animals treated with $L$ BMAA. It also suggests that the decline in volume in the motor cortex may be due to loss of GABAergic interneurons. Vucic et al. (2009) demonstrated in patients with ALS that intracortical inhibition mediated by inhibitory interneurons was significantly reduced, possibly by loss of interneurons.

In summary, we have described changes in rats treated with L-BMAA in glutamate and GABA levels which indicate that the neurotoxic effects of this amino acid could mimic important features of ALS in humans, such as hyperexcitability of cortical projecting neurons due to loss of GABAergic inhibition.

Taurine has gained great importance in recent years in the study of neurodegenerative diseases, since it plays an important protective role against glutamate-induced excitotoxicity, mainly through three mechanisms: inhibiting the influx of calcium into the cell induced by glutamate; inhibiting the opening of calcium channels and voltage-dependent calcium release from intracellular stores; and inhibiting internal glutamate induced apoptotic pathway (Menzie et al., 2014; Wu and Prentice, 2010). Recently, it was described in SOD1 transgenic mouse model of familial ALS, an increase of taurine in the brain in the later stages of the disease (Choi et al., 2009).

Our results show that, at least in the early times after administration of L-BMAA, taurine levels rise, possibly in response to excitotoxic processes caused by elevated levels of glutamate. Altered levels TauT has been proposed as a new disease marker for motoneuron under stress in ALS (Jung et al., 2013). However, the possible relationship between ALS and taurine require further study to confirm the possible therapeutic potential that may have this aminoacid to counteract the effects of excitotoxicity. 


\section{CONCLUSIONS}

In this paper we have shown that L-BMAA treated rats mimic several of the disorders presented by ALS patients, such as loss of hind limbs' muscle volume, which could imply muscular atrophy. On the other hand, these animals show motor cortex (M1 and M2 areas) thinning and ventricular enlargement. In addition, the trigeminal motor nucleus and the nucleus of the solitary tract, two bulbar nuclei involved in the motor control of swallowing, are affected by treatment with this neurotoxin. Moreover, these animals present alterations in the levels of glutamate, GABA and taurine in the motor cortex, which are consistent with the excitotoxic mechanism involved in neurodegeneration.

\section{ACKNOWLEDGEMENTS}

We wish to express our gratefulness to NMR - ESR Center (UCM), to Técnicas Geológicas CAI (UCM) and to Animal Facilities of Biological Science School (UCM) for their expert technical assistance. This work was supported by MINECO (PPT-300000-2009-11) and FUNDELA (Spanish Amiotrophic Lateral Sclerosis Foundation). 


\section{Bibliography:}

Agosta, F., Pagani, E., Rocca, M. A., Caputo, D., Perini, M., Salvi, F., Prelle, A., Filippi, M. (2007). "Voxel-based morphometry study of brain volumetry and diffusivity in amyotrophic lateral sclerosis patients with mild disability." Hum. Brain Mapp., 28(12), 1430-1438. doi: 10.1002/hbm.20364

Andjus, P. R., Bataveljić, D., Vanhoutte, G., Mitrecic, D., Pizzolante, F., Djogo, N., Nicaise, C., Gankam, K. F., Gangitano, C., Michetti, F., van der, L. A., Pochet, R., Bacic, G. (2009). "In vivo morphological changes in animal models of amyotrophic lateral sclerosis and Alzheimer's-like disease: MRI approach." Anat. Rec. (Hoboken.)., 292(12), 1882-1892. doi: 10.1002/ar.20995

Andreadou, E., Kapaki, E., Kokotis, P., Paraskevas, G. P., Katsaros, N., Libitaki, G., Petropoulou, O., Zis, V., Sfagos, C., Vassilopoulos, D. (2008). "Plasma glutamate and glycine levels in patients with amyotrophic lateral sclerosis." In Vivo, 22(1), 137-141.

Angenstein, F., Niessen, H. G., Goldschmidt, J., Vielhaber, S., Ludolph, A. C., Scheich, H. (2004). "Age-dependent changes in MRI of motor brain stem nuclei in a mouse model of ALS." Neuroreport, 15(14), 2271-2274.

Anneser, J. M., Chahli, C., Ince, P. G., Borasio, G. D., Shaw, P. J. (2004). "Glial proliferation and metabotropic glutamate receptor expression in amyotrophic lateral sclerosis." J. Neuropathol. Exp. Neurol, 63(8), 831840.

Arnold, A., Edgren, D. C., Palladino, V. S. (1953). "Amyotrophic lateral sclerosis; fifty cases observed on Guam." J. Nerv. Ment. Dis., 117(2), 135-139.

Bae, J. S., Simon, N. G., Menon, P., Vucic, S., Kiernan, M. C. (2013). "The puzzling case of hyperexcitability in amyotrophic lateral sclerosis." J. Clin. Neurol, 9(2), 65-74. doi: 10.3988/jcn.2013.9.2.65

Banack, S. A., Johnson, H. E., Cheng, R., Cox, P. A. (2007). "Production of the Neurotoxin BMAA by a Marine Cyanobacterium." Mar. Drugs., 5(4), 180196. doi:10.3390/md504180 
Basak, M., Erturk, M., Oflazoglu, B., Ozel, A., Yildiz, G. B., Forta, H. (2002). "Magnetic resonance imaging in amyotrophic lateral sclerosis." Acta Neurol. Scand., 105(5), 395-399. doi: 10.1034/j.1600-0404.2002.01321.x

Bataveljić, D., Djogo, N., Zupunski, L., Bajic, A., Nicaise, C., Pochet, R., Bacic, G., Andjus, P. R. (2009). "Live monitoring of brain damage in the rat model of amyotrophic lateral sclerosis." Gen. Physiol Biophys., $28 \mathrm{Spec}$ No:212-8., 212-218.

Berciano, J., Zarranz, J. J., Polo, J. M. (2003). "Enfermedad degenerativa espinal y espinocerebelosas." Neurología, Tercera ed. Madrid. Elsevier España, 689-695.

Boillèe, S., Peschanski, M., Junier, M. P. (2003). "The wobbler mouse: a neurodegeneration jigsaw puzzle." Mol. Neurobiol., 28(1), 65-106. doi: 10.1385/MN:28:1:65.

Bucher, S., Braunstein, K. E., Niessen, H. G., Kaulisch, T., Neumaier, M., Boeckers, T. M., Stiller, D., Ludolph, A. C. (2007). "Vacuolization correlates with spin-spin relaxation time in motor brainstem nuclei and behavioural tests in the transgenic G93A-SOD1 mouse model of ALS." Eur. J. Neurosci., 26(7), 1895-1901. doi: 10.1111/j.14609568.2007.05831.x

Choi, J. K., Kustermann, E., Dedeoglu, A., Jenkins, B. G. (2009). "Magnetic resonance spectroscopy of regional brain metabolite markers in FALS mice and the effects of dietary creatine supplementation." Eur. J. Neurosci., 30(11), 2143-2150. doi: 10.1111/j.1460-9568.2009.07015.x

Cosottini, M., Cecchi, P., Piazza, S., Pesaresi, I., Fabbri, S., Diciotti, S., Mascalchi, M., Siciliano, G., Bonuccelli, U. (2013). "Mapping cortical degeneration in ALS with magnetization transfer ratio and voxel-based morphometry." PLoS. One., 8(7), e68279. doi: 10.1371/journal.pone.0068279

Cox, P. A., Banack, S. A., Murch, S. J. (2003). "Biomagnification of cyanobacterial neurotoxins and neurodegenerative disease among the Chamorro people of Guam." Proc. Natl. Acad. Sci. U. S. A., 100(23), 13380-13383. doi: 10.1073/pnas.2235808100

Cox, P. A., Banack, S. A., Murch, S. J., Rasmussen, U., Tien, G., Bidigare, R. R., Metcalf, J. S., Morrison, L. F., Codd, G. A., Bergman, B. (2005). 
"Diverse taxa of cyanobacteria produce beta-N-methylamino-L-alanine, a neurotoxic amino acid." Proc. Natl. Acad. Sci. U. S. A., 102(14), 50745078. doi: 10.1073/pnas.0501526102

Cox, P. A., Sacks, O. W. (2002). "Cycad neurotoxins, consumption of flying foxes, and ALS-PDC disease in Guam." Neurology, 58(6), 956-959.

de Munck, E., Muñoz-Sáez, E., Miguel, B. G., Solas, M. T., Ojeda, I., Martínez, A., Gil, C., Arahuetes, R. M. (2013). "beta-N-methylamino-I-alanine causes neurological and pathological phenotypes mimicking Amyotrophic Lateral Sclerosis (ALS): the first step towards an experimental model for sporadic ALS." Environ. Toxicol. Pharmacol., 36(2), 243-255. doi: 10.1016/j.etap.2013.04.007

Dunlop, R. A., Cox, P. A., Banack, S. A., Rodgers, K. J. (2013). "The NonProtein Amino Acid BMAA Is Misincorporated into Human Proteins in Place of I-Serine Causing Protein Misfolding and Aggregation." PLoS. One., 8(9), e75376. doi: 10.1371/journal.pone.0075376

Evans, M. C., Couch, Y., Sibson, N., Turner, M. R. (2013). "Inflammation and neurovascular changes in amyotrophic lateral sclerosis." Mol. Cell Neurosci., 53, 34-41. doi: 10.1016/j.mcn.2012.10.008

Filippini, N., Douaud, G., Mackay, C. E., Knight, S., Talbot, K., Turner, M. R. (2010). "Corpus callosum involvement is a consistent feature of amyotrophic lateral sclerosis." Neurology, 75(18), 1645-1652. doi: 10.1212/WNL.0b013e3181fb84d1

Foerster, B. R., Callaghan, B. C., Petrou, M., Edden, R. A., Chenevert, T. L., Feldman, E. L. (2012). "Decreased motor cortex gamma-aminobutyric acid in amyotrophic lateral sclerosis." Neurology, 78(20), 1596-1600. doi: 10.1212/WNL.0b013e3182563b57

Foerster, B. R., Pomper, M. G., Callaghan, B. C., Petrou, M., Edden, R. A., Mohamed, M. A., Welsh, R. C., Carlos, R. C., Barker, P. B., Feldman, E. L. (2013). "An imbalance between excitatory and inhibitory neurotransmitters in amyotrophic lateral sclerosis revealed by use of 3-T proton magnetic resonance spectroscopy." JAMA Neurol, 70(8), 10091016. doi: 10.1001/jamaneurol.2013.234

Fogal, B., Li, J., Lobner, D., McCullough, L. D., Hewett, S. J. (2007). "System $x(c)$ - activity and astrocytes are necessary for interleukin-1 beta- 
mediated hypoxic neuronal injury." J. Neurosci., 27(38), 10094-10105. doi: 10.1523/JNEUROSCI.2459-07.2007

Grosskreutz, J., Kaufmann, J., Fradrich, J., Dengler, R., Heinze, H. J., Peschel, T. (2006). "Widespread sensorimotor and frontal cortical atrophy in Amyotrophic Lateral Sclerosis." BMC. Neurol., 6, 17. doi:10.1186/14712377-6-17

Guegan, C., Przedborski, S. (2003). "Programmed cell death in amyotrophic lateral sclerosis." J. Clin. Invest, 111(2), 153-161. doi:10.1172/JCI17610

Hall, E. D., Oostveen, J. A., Gurney, M. E. (1998). "Relationship of microglial and astrocytic activation to disease onset and progression in a transgenic model of familial ALS." Glia, 23(3), 249-256.

Hecht, M. J., Fellner, F., Fellner, C., Hilz, M. J., Neundorfer, B., Heuss, D. (2002). "Hyperintense and hypointense MRI signals of the precentral gyrus and corticospinal tract in ALS: a follow-up examination including FLAIR images." J. Neurol. Sci., 199(1-2), 59-65. DOI: http://dx.doi.org/10.1016/S0022-510X(02)00104-1

Hensley, K., Mhatre, M., Mou, S., Pye, Q. N., Stewart, C., West, M., Williamson, K. S. (2006). "On the relation of oxidative stress to neuroinflammation: lessons learned from the G93A-SOD1 mouse model of amyotrophic lateral sclerosis." Antioxid. Redox. Signal., 8(11-12), 2075-2087. doi:10.1089/ars.2006.8.2075

Holtcamp, W. (2012). "The emerging science of BMAA: do cyanobacteria contribute to neurodegenerative disease?" Environ. Health Perspect., 120(3), A110-A116. doi: 10.1289/ehp.120-a110

Jiang, L., Eriksson, J., Lage, S., Jonasson, S., Shams, S., Mehine, M., Ilag, L. L., Rasmussen, U. (2014). "Diatoms: A Novel Source for the Neurotoxin BMAA in Aquatic Environments." PLoS. One., 9(1), e84578. doi: 10.1371/journal.pone.0084578

Jung, M. K., Kim, K. Y., Lee, N. Y., Kang, Y. S., Hwang, Y. J., Kim, Y., Sung, J. J., McKee, A., Kowall, N., Lee, J., Ryu, H. (2013). "Expression of taurine transporter (TauT) is modulated by heat shock factor 1 (HSF1) in motor neurons of ALS." Mol. Neurobiol., 47(2), 699-710. doi: 10.1007/s12035012-8371-9 
Karamyan, V. T., Speth, R. C. (2008). "Animal models of BMAA neurotoxicity: a critical review." Life Sci., 82(5-6), 233-246. doi: 10.1016/j.Ifs.2007.11.020

Kato, S., Shaw, P., Wood-Allum, C., Leigh, N. P., Shaw, C. E. (2014). "Amyotrophic lateral sclerosis." Neurodegeneration: the molecular pathology of dementia and movement disorders. $1^{\mathrm{a}}$ ed. Basel: Blackwell Publishing, 350-371.

Kolind, S., Sharma, R., Knight, S., Johansen-Berg, H., Talbot, K., Turner, M. R. (2013). "Myelin imaging in amyotrophic and primary lateral sclerosis." Amyotroph. Lateral. Scler. Frontotemporal. Degener., 14(7-8), 562-573. doi: 10.3109/21678421.2013.794843

Kollewe, K., Korner, S., Dengler, R., Petri, S., Mohammadi, B. (2012). "Magnetic resonance imaging in amyotrophic lateral sclerosis." Neurol Res. Int., 2012, 608501. doi: 10.1155/2012/608501

Kurland, L. T., Mulder, D. W. (1954 a). "Epidemiologic investigations of amyotrophic lateral sclerosis. I. Preliminary report on geographic distribution and special reference to the Mariana Islands, including clinical and pathologic observations." Neurology, 4(6), 438-448.

Kurland, L. T., Mulder, D. W. (1954 b). "Epidemiologic investigations of amyotrophic lateral sclerosis. I. Preliminary report on geographic distribution, with special reference to the Mariana Islands, including clinical and pathologic observations." Neurology, 4(5), 355-378.

Leigh, P. N., Meldrum, B. S. (1996). "Excitotoxicity in ALS." Neurology, 47(6 Suppl 4), S221-S227.

Lillo, P., Mioshi, E., Burrell, J. R., Kiernan, M. C., Hodges, J. R., Hornberger, M. (2012). "Grey and White Matter Changes across the Amyotrophic Lateral Sclerosis-Frontotemporal Dementia Continuum." PLoS. One., 7(8), e43993. doi: 10.1371/journal.pone.0043993

Liu, X. Q., Rush, T., Ciske, J., Lobner, D. (2010). "Selective death of cholinergic neurons induced by beta-methylamino-L-alanine." Neuroreport., 21(1), 55-58. doi: 10.1097MNR.0b013e328333dfd5

Liu, X., Rush, T., Zapata, J., Lobner, D. (2009). "beta-N-methylamino-I-alanine induces oxidative stress and glutamate release through action on system Xc(-)." Exp. Neurol., 217(2), 429-433. doi: 10.1016/j.expneurol.2009.04.002 
Lobner, D., Piana, P. M., Salous, A. K., Peoples, R. W. (2007). "Beta-Nmethylamino-L-alanine enhances neurotoxicity through multiple mechanisms." Neurobiol. Dis., 25(2), 360-366. doi: 10.1016/j.nbd.2006.10.002

Malessa, S., Leigh, P. N., Bertel, O., Sluga, E., Hornykiewicz, O. (1991). "Amyotrophic lateral sclerosis: glutamate dehydrogenase and transmitter amino acids in the spinal cord." J. Neurol Neurosurg. Psychiatry, 54(11), 984-988.

Martin, L. J., Chang, Q. (2012). "Inhibitory synaptic regulation of motoneurons: a new target of disease mechanisms in amyotrophic lateral sclerosis." Mol. Neurobiol., 45(1), 30-42. doi: 10.1007/s12035-011-8217-x

Menzie, J., Pan, C., Prentice, H., Wu, J. Y. (2014). "Taurine and central nervous system disorders." Amino. Acids, 46(1), 31-46. doi: 10.1007/s00726-012$1382-z$

Mezzapesa, D. M., Ceccarelli, A., Dicuonzo, F., Carella, A., De Caro, M. F., Lopez, M., Samarelli, V., Livrea, P., Simone, I. L. (2007). "Whole-brain and regional brain atrophy in amyotrophic lateral sclerosis." AJNR Am. J. Neuroradiol., 28(2), 255-259.

Mezzapesa, D. M., D'Errico, E., Tortelli, R., Distaso, E., Cortese, R., Tursi, M., Federico, F., Zoccolella, S., Logroscino, G., Dicuonzo, F., Simone, I. L. (2013). "Cortical thinning and clinical heterogeneity in amyotrophic lateral sclerosis." PLoS. One., 8(11), e80748. doi: 10.1371/journal.pone.0080748

Mitchell, J. D., Borasio, G. D. (2007). "Amyotrophic lateral sclerosis." Lancet, 369(9578), 2031-2041. doi:10.1016/S0140-6736(07)60944-1

Mulder, D. W., Kurland, L. T. (1987). "Motor neuron disease: epidemiologic studies." Adv. Exp. Med. Biol., 209, 325-332.

Murch, S. J., Cox, P. A., Banack, S. A. (2004). "A mechanism for slow release of biomagnified cyanobacterial neurotoxins and neurodegenerative disease in Guam." Proc. Natl. Acad. Sci. U. S. A., 101(33), 12228-12231. doi: 10.1073/pnas.0404926101

Murphy, T. H., Miyamoto, M., Sastre, A., Schnaar, R. L., Coyle, J. T. (1989). "Glutamate toxicity in a neuronal cell line involves inhibition of cystine 
transport leading to oxidative stress." Neuron, 2(6), 1547-1558. DOI: http://dx.doi.org/10.1016/0896-6273(89)90043-3

Murphy, T. H., Schnaar, R. L., Coyle, J. T. (1990). "Immature cortical neurons are uniquely sensitive to glutamate toxicity by inhibition of cystine uptake." FASEB J., 4(6), 1624-1633.

Myers, T. G., Nelson, S. D. (1990). "Neuroactive carbamate adducts of beta-Nmethylamino-L-alanine and ethylenediamine. Detection and quantitation under physiological conditions by 13C NMR." J. Biol. Chem., 265(18), 10193-10195.

Niessen, H. G., Angenstein, F., Sander, K., Kunz, W. S., Teuchert, M., Ludolph, A. C., Heinze, H. J., Scheich, H., Vielhaber, S. (2006). "In vivo quantification of spinal and bulbar motor neuron degeneration in the G93A-SOD1 transgenic mouse model of ALS by T2 relaxation time and apparent diffusion coefficient." Exp. Neurol., 201(2), 293-300. doi: 10.1016/j.expneurol.2006.04.007

Nihei, K., McKee, A. C., Kowall, N. W. (1993). "Patterns of neuronal degeneration in the motor cortex of amyotrophic lateral sclerosis patients." Acta Neuropathol., 86(1), 55-64.

Nishihira, Y., Tan, C. F., Onodera, O., Toyoshima, Y., Yamada, M., Morita, T., Nishizawa, M., Kakita, A., Takahashi, H. (2008). "Sporadic amyotrophic lateral sclerosis: two pathological patterns shown by analysis of distribution of TDP-43-immunoreactive neuronal and glial cytoplasmic inclusions." Acta Neuropathol., 116(2), 169-182. doi: 10.1007/s00401008-0385-z

Nunn, P. B., Seelig, M., Zagoren, J. C., Spencer, P. S. (1987). "Stereospecific acute neuronotoxicity of 'uncommon' plant amino acids linked to human motor-system diseases." Brain Res., 410(2), 375-379. doi: 10.1016/00068993(87)90342-8

Pablo, J., Banack, S. A., Cox, P. A., Johnson, T. E., Papapetropoulos, S., Bradley, W. G., Buck, A., Mash, D. C. (2009). "Cyanobacterial neurotoxin BMAA in ALS and Alzheimer's disease." Acta Neurol. Scand., 120(4), 216-225. doi: 10.1111/j.1600-0404.2008.01150.x

Paxinos, G., Watson, C. (2005). "The Rat Brain in Stereotaxic Coordinates. 5th edition." Ed. Elservier Academic Press. 
Perry, T. L., Hansen, S., Jones, K. (1987). "Brain glutamate deficiency in amyotrophic lateral sclerosis." Neurology, 37(12), 1845-1848.

Prell, T., Peschel, T., Hartung, V., Kaufmann, J., Klauschies, R., Bodammer, N., Kollewe, K., Dengler, R., Grosskreutz, J. (2013). "Diffusion tensor imaging patterns differ in bulbar and limb onset amyotrophic lateral sclerosis." Clin. Neurol Neurosurg., 115(8), 1281-1287. doi: 10.1016/j.clineuro.2012.11.031

Provenzale, J. (2004). "Dementias and degenerative disorders." Diagnostic Imaging Brain. $1^{\mathrm{a}}$ ed. Salt Lake City: Amirsys, 86-89.

Reiner, A., Medina, L., Figueredo-Cardenas, G., Anfinson, S. (1995). "Brainstem motoneuron pools that are selectively resistant in amyotrophic lateral sclerosis are preferentially enriched in parvalbumin: evidence from monkey brainstem for a calcium-mediated mechanism in sporadic ALS." Exp. Neurol, 131(2), 239-250.

Robberecht, W. (2000). "Oxidative stress in amyotrophic lateral sclerosis." J. Neurol., 247(Suppl 1), 1-6. doi: 10.1007/s004150050551

Robberecht, W., Philips, T. (2013). "The changing scene of amyotrophic lateral sclerosis." Nat. Rev. Neurosci., 14(4), 248-264. doi: 10.1038/nrn3430

Rodgers, K. J. (2014). "Non-protein amino acids and neurodegeneration: The enemy within." Exp. Neurol, 253C, 192-196. doi: 10.1016/j.expneurol.2013.12.010

Rodgers, K., Dunlop, R. (2011). "The cyanobacteria-derived BMAA can be incorporated into cell proteins and could thus be an environmental trigger for ALS and other neurological diseases associated with protein misfolding." Amyotrophic Lateral Sclerosis, 12(Suppl. 1), 22. doi: 10.3109/17482968.2011.615501

Ross, S. M., Seelig, M., Spencer, P. S. (1987). "Specific antagonism of excitotoxic action of 'uncommon' amino acids assayed in organotypic mouse cortical cultures." Brain Res., 425(1), 120-127.

Ross, S. M., Spencer, P. S. (1987). "Specific antagonism of behavioral action of "uncommon" amino acids linked to motor-system diseases." Synapse, 1(3), 248-253. 
Spencer, P. S., Hugon, J., Ludolph, A., Nunn, P. B., Ross, S. M., Roy, D. N., Schaumburg, H. H. (1987 a). "Discovery and partial characterization of primate motor-system toxins." Ciba Found. Symp., 126, 221-238.

Spencer, P. S., Nunn, P. B., Hugon, J., Ludolph, A. C., Ross, S. M., Roy, D. N., Robertson, R. C. (1987 b). "Guam amyotrophic lateral sclerosisparkinsonism-dementia linked to a plant excitant neurotoxin." Science, 237(4814), 517-522.

Spreux-Varoquaux, O., Bensimon, G., Lacomblez, L., Salachas, F., Pradat, P. F., Le Forestier, N., Marouan, A., Dib, M., Meininger, V. (2002). "Glutamate levels in cerebrospinal fluid in amyotrophic lateral sclerosis: a reappraisal using a new HPLC method with coulometric detection in a large cohort of patients." J. Neurol Sci., 193(2), 73-78. doi: http://dx.doi.org/10.1016/S0022-510X(01)00661-X

Turner, B. J., Talbot, K. (2008). "Transgenics, toxicity and therapeutics in rodent models of mutant SOD1-mediated familial ALS." Prog. Neurobiol., 85(1), 94-134. doi: 10.1016/j.pneurobio.2008.01.001

Turner, M. R., Cagnin, A., Turkheimer, F. E., Miller, C. C., Shaw, C. E., Brooks, D. J., Leigh, P. N., Banati, R. B. (2004). "Evidence of widespread cerebral microglial activation in amyotrophic lateral sclerosis: an [11C](R)-PK11195 positron emission tomography study." Neurobiol. Dis., 15(3), 601-609. doi: 10.1016/j.nbd.2003.12.012

Vega, A., Bell, E. A. (1967). "Alpha-amino-beta-methilaminopropionic acid, a new amino acid from seeds of Cycas circinalis." Phytochemistry, 6, 759762.

Vega, A., Bell, E. A., Nunn, P. B. (1968). "The preparation of L- and D- alphaamino-beta-methylaminopropionic acid and the identification of the compound isolated from Cycas circinalis as the L-isomer." Phytochemistry, 7, 1885-1887.

Verstraete, E., van den Heuvel, M. P., Veldink, J. H., Blanken, N., Mandl, R. C., Hulshoff Pol, H. E., van den Berg, L. H. (2010). "Motor network degeneration in amyotrophic lateral sclerosis: a structural and functional connectivity study." PLoS. One., 5(10), e13664. doi: 10.1371/journal.pone.0013664 
Vucic, S., Cheah, B. C., Kiernan, M. C. (2009). "Defining the mechanisms that underlie cortical hyperexcitability in amyotrophic lateral sclerosis." Exp. Neurol, 220(1), 177-182. doi: 10.1016/j.expneurol.2009.08.017

Walker, A. K., Atkin, J. D. (2011). "Stress signaling from the endoplasmic reticulum: A central player in the pathogenesis of amyotrophic lateral sclerosis." IUBMB. Life, 63(9), 754-763. doi: 10.1002/iub.520

Weydt, P., Weiss, M. D., Moller, T., Carter, G. T. (2002). "Neuro-inflammation as a therapeutic target in amyotrophic lateral sclerosis." Curr. Opin. Investig. Drugs, 3(12), 1720-1724.

Wijesekera, L. C., Leigh, P. N. (2009). "Amyotrophic lateral sclerosis." Orphanet. J. Rare. Dis., 4:3, 3. doi: 10.1186/1750-1172-4-3

Wu, J. Y., Prentice, H. (2010). "Role of taurine in the central nervous system." J. Biomed. Sci., 17 Suppl 1:S1. doi: 10.1186/1423-0127-17-S1-S1

Zang, D. W., Yang, Q., Wang, H. X., Egan, G., Lopes, E. C., Cheema, S. S. (2004). "Magnetic resonance imaging reveals neuronal degeneration in the brainstem of the superoxide dismutase 1 transgenic mouse model of amyotrophic lateral sclerosis." Eur. J. Neurosci., 20(7), 1745-1751. doi: 10.1111/j.1460-9568.2004.03648.x

Zhang, L., Ulug, A. M., Zimmerman, R. D., Lin, M. T., Rubin, M., Beal, M. F. (2003). "The diagnostic utility of FLAIR imaging in clinically verified amyotrophic lateral sclerosis." J. Magn Reson. Imaging, 17(5), 521-527. doi: 10.1002/jmri.10293 


\section{Highlights:}

- $\quad$ Rats treated with L-BMAA have progressive muscular loss of volume.

- L-BMAA causes thinning of the motor cortex and the enlargement of the lateral ventricles.

- Animals treated with L-BMAA show damage in motor nuclei involved in swallowing.

- L-BMAA causes deregulation of the glutamatergic and GABAergic systems in the long term. 